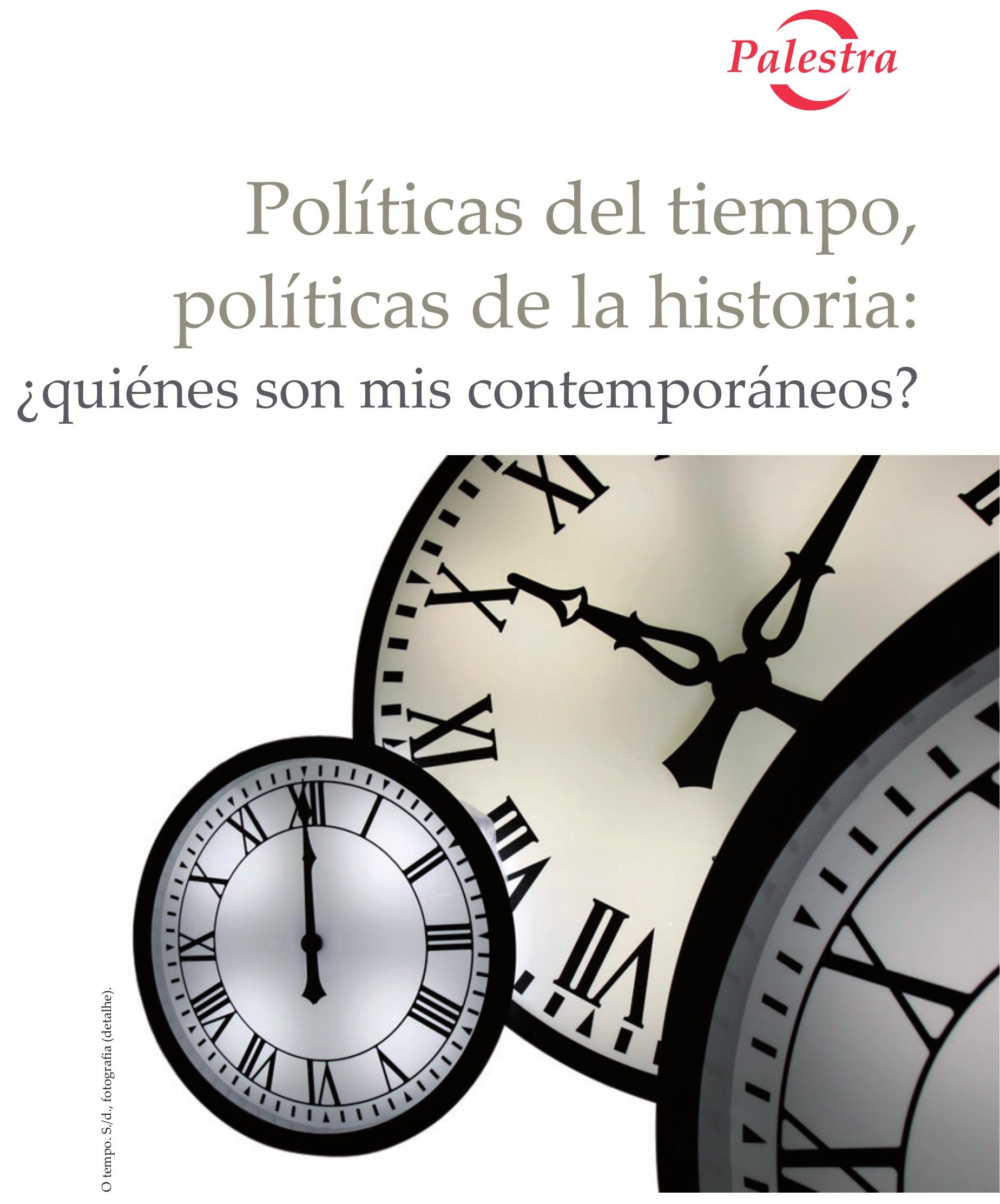

María Inés Mudrovcic

Doutora em Filosofia pela Universidade de Buenos Aires. Professora da Universidad Nacional de Comahue (UNCo). Pesquisadora do Conicet. Organizadora, entre outros livros, de En busca del tiempo pasado: temporalidad, historia y memoria. México: Siglo XXI, 2013. mmudrovcic@gmail.com 


\section{Políticas del tiempo, políticas de la historia: ¿quiénes son mis contemporáneos?*}

Time politics, history politics: who are my contemporaries?

María Inés Mudrovcic

\section{RESUMEN}

En este trabajo intento abordar la importancia que posee el análisis del presente para una teoría del tiempo histórico. A tal fin, abordo la noción de "políticas del tiempo" como actos que realizamos en el presente y que determinan quiénes lo habitan. En primer lugar, intento mostrar cómo en el siglo XIX y, en Occidente, el presente comenzó a ser experimentado como “contemporáneo". En segundo lugar, discuto los presupuestos que subyacen a esta experiencia de lo contemporáneo y de qué forma produce una discriminación sincrónica (los "primitivos" o "salvajes" de la antropología) y diacrónica (los "muertos" del pasado histórico).

PALABRAS-CLAVE: presente; tiempo histórico; políticas del tiempo.

\begin{abstract}
In this paper I intend to address the importance of the analysis of the present for a theory of historical time. To that end, I approachthe notion of "politics of time" as actsthatwecarryout in the present and that determine whoinhabitsit. First, I try to show how in thenineteenthcentury and, in the West, the presentbeganto be experienced as "contemporary". Secondly, I discussthe presuppositions thatunderliethisexperience of the contemporary and howit produces a synchronicdiscrimination (the "primitives" or "savages" of anthropology) and diachronic (the "dead" of thehistoricalpast)
\end{abstract}

KEYWORDS: present; historical time; politics of time.
* Conferencia dictada en el IV Congreso Internacional de Filosofía de la Historia. Buenos Aires, 8 de noviembre de 2017.

\section{Apresentação da autora}

Ao longo das últimas décadas, foram diversos os avanços epistemológicos e programáticos no campo da teoria da história e da filosofia da história, a ponto de não hesitarmos mais em falarmos em consolidação disciplinar. Reconhecemos problemas e abordagens específicos, assistimos a uma profusão de objetos de investigação e verificamos uma inserção cada vez mais expressiva de componentes curriculares que abrigam reflexões teóricas. Parecemos cada vez mais distantes da velha máxima segundo a qual a teorização deveria servir ao historiador exclusivamente como um arsenal de instrumentos conceituais e analíticos prontos a serem empregados na pesquisa empírica, que era, afinal, o que importava em nosso trabalho.

Nada disso teria sido possível, ao menos entre nós, sem a formação de redes de pesquisadores envolvendo nossos vizinhos latino-americanos. Apesar das visíveis distinções resultantes de sistemas universitários e curriculares particulares, há um crescente compartilhamento de problemas, 
de temas de pesquisa, de diálogos estabelecidos em congressos, colóquios e seminários e de publicações em livros e revistas especializadas. Uma comunidade continental de investigadores interessados nas condições de possibilidade da escrita da história tem deixado de ser um mero horizonte desejável para se tornar, cada vez mais, uma realidade concreta.

A obra de María Inés Mudrovcic é uma das referências fundamentais do processo que acabo de descrever sucintamente. Intelectual que desfruta de grande reconhecimento acadêmico, diretora do Centro de Investigación en Filosofia de las Ciencias Sociales y Humanidades e professora titular de Filosofia da História da Universidad Nacional de Comahue, Mudrovcic tem uma extensa e sólida produção que transita por temas diversos, sempre com distinta originalidade e profundidade. De seu estudo já clássico sobre Voltaire e o iluminismo ${ }^{1}$ até as investigações mais recentes sobre as políticas do tempo histórico ${ }^{2}$ - tema do texto que publicamos nesta edição da ArtCultura-, passando ainda por incursões sobre as relações entre trauma e história, memória e narrativa ${ }^{3}$, ela produziu uma reflexão teórica de largo escopo a partir de marcas e tonalidades próprias, oriundas especialmente do recente passado traumático argentino, cujo peso do regime ditatorial nos soa tragicamente familiar. Nesta chave interpretativa, deve-se enfatizar, portanto, que seus escritos - compreendendo também um expressivo números de artigos e capítulos de livros - indicam a vitalidade de um pensamento que dialoga com a produção internacional sem abandonar os esforços cada vez mais urgentes de pensar as epistemologias do Sul. O público brasileiro tem se beneficiado, já há algum tempo, de sua presença constante em eventos acadêmicos em nosso país e é possível conhecê-la um pouco mais por intermédio de uma valiosa entrevista publicada, em 2017, nas páginas da revista Tempo e Argumento. ${ }^{4}$

Destacada a indiscutível relevância da autora, cabe-nos reafirmar o grande orgulho em vê-la publicada em nossa ArtCultura.

\section{Alexandre de Sá Avelar ${ }^{5}$}

\section{$* * *$}

¿Qué es el presente?, ¿quiénes lo habitan?, ¿quiénes son mis contemporáneos? Tanto teóricos como filósofos de la historia se han ocupado cada vez en mayor medida del problema del tiempo histórico, pero, sin embargo, la cuestión acerca de qué es o cuál es la estructura temporal del presente casi no se ha puesto en discusión. Aun cuando la noción de presente siempre estuvo implicada en el quehacer historiográfico y es el punto ciego de la historia, no es sino hasta hace muy poco que comienza a tener un lugar central, sobre todo, a partir de las obras François Hartog ${ }^{6}$ y Hans Gumbrecht. ${ }^{7}$ Pensemos, por ejemplo, en el clásico libro de Reinhart Koselleck, Futuro pasado", en el que las dos categorías centrales son "espacio de experiencia" y "horizonte de expectativas" y el presente parece reducirse a una simple intersección o articulación entre ambas, sin densidad alguna. Pasado y futuro han sido tradicionalmente las dos categorías temporales que han circunscripto a la historia, en desmedro del presente.

Es difícil asir el presente. En el intento por tratar de darle un contorno a lo que entendemos por presente, no lo pienso como algo que experimentamos pasivamente, sino como resultado de actos que realizamos con el tiempo. Parafraseando a John Austin, ${ }^{9}$ la idea es pensar cómo hacemos
${ }^{1}$ MUDROVCIC, María Inés. Voltaire, el Iluminismo y la Historia. Buenos Aires: Fundec, 1996.

${ }^{2}$ MUDROVCIC, María Inés e RABOTNIKOF, Nora. En busca del pasado perdido: temporalidad, historia y memoria. México: Siglo XXI, 2013.

${ }^{3}$ MUDROVCIC, María Inés. Historia, narración y memória: los debates actuales em filosofía de la historia. Madrid: Akal, 2009, e Pasados em conflicto: representación, mito y memoria. Buenos Aires: Prometeo, 2009;

${ }^{4}$ História do tempo presente e América Latina: Argentina uma entrevista com María Inés Mudrovcic. Tempo e Argumento, v. 9, n. 21, Florianópolis, maioago. 2017. Entrevistadoras: Camila Serafim Daminelli e Elisangela da Silva Machieski.

${ }^{5}$ Doutor em História pela Universidade Federal Fluminense (UFF). Professor dos cursos de graduação e pós-graduação em História da Universidade Federal de Uberlândia (UFU). Pesquisador do CNPq. Coorganizador, entre outros livros, de Afirmação da história como ciência no século XX: de ArletteFarge a Robert Mandrou. Petrópolis: Vozes, 2016. alexandre.avelar@uol.com.br

${ }^{6}$ HARTOG, François. Régimes d'historicité : présentisme et expériences du temps. Paris: Éditions du Seuil, 2003.

${ }^{7}$ GUMBRECHT, Hans U. Lento presente: sintomatología del nuevo tiempo histórico. Madrid: Escobar y Mayo Editores S. L., 2010.

${ }^{8}$ KOSELLECK, Reinhart. Futuro pasado: para una semántica de los tiempos históricos. Barcelona: Paidós, 1993, p. 333 y 358.

${ }^{9}$ AUSTIN, John Langshaw. How to do things with words? Oxford: Clarendon Press, 1962. 
${ }^{10}$ DAWKINS, Richard. Los locos románticos del Brexit. 2017. Disponible en <http:// elpais.com/elpais/2017/04/02/ opinion/1491143414_963850. html>. Acceso al 01 nov. 2017.

${ }^{11}<$ https://shop.donaldjtrump. com/>. Acceso al 15 out.2017. cosas con el tiempo, a esa operación sobre el tiempo yo la denomino "políticas del tempo". Las políticas del tiempo cualifican al pasado, al presente y al futuro. Un tiempo cronológico, meramente cuantitativo, que se constituya en parámetro desinteresado y objetivo de las actividades humanas es impensable y, aun cuando lo fuese, sería resultado de lo que aquí denomino "políticas del tiempo". El término "política" no debe ser entendido como sinónimo de ideología o de valores políticos, sino como una forma de autoridad que, en el caso específico del tiempo, determina qué o quiénes pertenecen al presente, quienes son mis contemporáneos. Las políticas del tiempo consisten en un conjunto de operaciones que, a la vez que sancionan lo que es propio o característico del presente, construyen un "otro" excluyéndolo diacrónica o sincrónicamente de ese presente, el otro es anacrónico. El otro no es mi contemporáneo.

Voy a dar algunos ejemplos. El 10 de diciembre de 2016 el gobierno argentino crea el Ministerio de Modernización el que, entre otras cuestiones, se propone “Diseñar, proponer y coordinar las políticas de transformación y modernización del Estado". Su ministro acaba de expresar, hace pocos días atrás, que habían recibido un estado "arcaico", propio del "siglo XIX". El científico inglés Richard Dawkinsen una columna que publicara el diario El País el 2 de abril de 2017 confiesa abiertamente el "desprecio" que le provoca "la mezquindad moral, espiritual, política e intelectual de los británicos que votaron a favor del Brexit en el referéndum" (2015). En un intento de explicación cuasi freudiano, Dawkins atribuye el voto pro Brexit, de los que él denomina "gerontobrexiteros", a la nostalgia de la "juventud perdida". ${ }^{10}$ En su campaña para llegar a la presidencia de los EEUU en 2016, Donald Trump reflotó un viejo eslogan que Ronald Reagan utilizara en la suya en 1980: “Make America great again!”. El lema adquirió tanto éxito entre sus seguidores que permitió abrir una tienda on line para recaudar fondos para la campaña con la venta de los más disímiles objetos - desde una taza hasta un sombrero - con el eslogan impreso. ${ }^{11}$ En uno de los últimos spots televisivos de la última campaña política en Argentina, el candidato Lousteau anuncia que "hay que dejar el pasado bien atrás" y que hay que elegir "avanzar". Éstos no son sino ejemplos de lo que en este trabajo denomino "políticas del tiempo".

Las políticas del tiempo son acciones sobre el tiempo, más o menos reconocidas como tales, que constituyen a un "otro" al excluirlo del presente. Es así que, en Argentina, un estado "moderno" se erige frente al estado "obsoleto y arcaico" heredado del pasado. La arena política simplifica generacionalmente el resultado expulsando del presente a los votantes pro Brexit como "viejos" nostálgicos. El eslogan de Trump intenta recuperar los "good old days" que, según la mitología norteamericana, fueron un tiempo en los que nadie dudaba de la superioridad y bonanza americana. La América grandiosa del pasado se opone a la América insignificante y débil del presente que encontrara Trump. El estado obsoleto, los viejos nostálgicos, la América débil, y el pasado que atrasa del político argentino son "anacrónicos", es decir, están fuera del presente. Aun cuando todos los votantes del Brexit vivan en un mismo presente cronológico, no todos "pertenecen" al mismo: los que votaron a favor son los "gerontes del pasado". Según funcionarios del gobierno argentino, la estructura del Estado actual no es la adecuada para el presente "moderno". El "pasado glorioso" de América es, para Trump, más propio del presente. Y a la inversa para el político argentino, el pasado que atrasa no es propio de un presente que 
debe dirigirse firme a un futuro. La pregunta es: ¿por qué discriminamos así?, ¿cómo creamos este tipo de alteridades temporales?

Originalmente, "contemporáneo", que deriva del latín cum tempo, significó ocurrir o vivir en el mismo período de tiempo. Es en este sentido que deben entenderse frases tales como "Aristóteles fue contemporáneo de Platón y Alejandro Magno" o "Churchill fue contemporáneo de Hitler". Es decir, el adjetivo contemporáneo relaciona acontecimientos que ocurren en un mismo tiempo. Este sentido, aparentemente casi cronológico del adjetivo contemporáneo, y que se conserva hasta nuestros días, aparece en la lengua francesa alrededor de 1475. Un claro ejemplo de este uso está en los Pensées (1670) de Pascal cuando establece como principio epistemológico que se debe desconfiar de toda historia que no haya sido escrita por aquellos que fueron contemporáneos a los acontecimientos que se narran.

Sin embargo, durante el siglo XIX, primero en Francia y luego en el resto de Europa, se consolida otra forma de entender la contemporaneidad. Lo contemporáneo va a cualificar, ahora, al presente. Es decir, contemporáneos son aquellos que comparten el presente postrevolucionario. Contemporáneo ya no alude solamente a una mera sincronización temporal (compartir un mismo tiempo), sino que la sincronización se acota al presente de las generaciones "vivas" que habitaban Europa y EEUU luego de la Revolución Francesa.

El siglo XIX francés delimita su presente, al que llama "contemporáneo"- al establecer que la Revolución instaura una barrera con lo que se llamó "Antiguo Régimen". La expresión Ancien régime, conocida por los revolucionarios franceses y popularizada por Tocqueville ${ }^{12}$ y Taine ${ }^{13}$ designa al "régimen feudal", pasado que, para ellos, era muy próximo en el tiempo, pero que ambos consideran ya ido totalmente. Para Tocqueville, el sólo hecho de acuñar dicho nombre abre un "abismo" entre el pasado prerevolucionario y su futuro: “Los franceses hicieron, en 1789, el más grande esfuerzo que haya sido hecho por un pueblo para desgarrar su historia en dos partes, por decirlo de alguna manera, y abrir un abismo entre su pasado y su futuro".${ }^{14}$ La Revolución es lo que separa al Antiguo Régimen de ese presente que se concibe como totalmente diferente. El período contemporáneo es ese novísimo presente (neueste) que comparten los europeos que viven luego de la Revolución Francesa. Tocqueville expresa claramente esta idea en 1856: "Por lo tanto me pareció que la forma apropiada de estudiar la Revolución era olvidar, por un momento, la Francia que tenemos ante nosotros, para examinar, en su tumba, la Francia que se ha ido". ${ }^{15}$

Francia primero y Europa, después, es decir, lo que desde 1890 iba a ser denominado como "Occidente", comienzan a constituirse en el estándar temporal a partir del cual se comienzan a "medir" el resto de las naciones. Como un "naturalista" frente a un insecto, Taine intentará explicar la situación presente por la que atraviesa Francia describiendo los estadios que, desde el Antiguo Régimen, pasando por la Revolución, conducen al Régimen Moderno. Igual que para Tocqueville, la Revolución interpone una barrera infranqueable entre el pasado ya ido del Antiguo Régimen y la Francia contemporánea. Es un pasado que pertenece sólo a la "vieja" Francia. El presente contemporáneo se afirma excluyendo al pasado. Los muertos pertenecen al pasado. La Francia que Tocqueville tiene ante sí pertenece a un presente que se experimenta como totalmente distinto del pasado. Es un presente que se distingue de su pasado como lo vivo de lo muerto. El presente contemporáneo es compartido por los vivos que miran

${ }^{12}$ TOCQUEVILLE, Alexis de (1856). L'Ancien régime et la révolution. Paris: Gallimard, 1952.

${ }^{13}$ TAINE, Hippolyte. Les origines de la France contemporaine. Paris: Hachette, 1875.

${ }^{14}$ TOCQUEVILLE, Alexis de, op. cit., p. 1.

${ }^{15}$ Idem, ibidem. 
${ }^{16}<$ https://greenwichmeantime. com/info/conference $>$. Acceso al 01 nov. 2017.
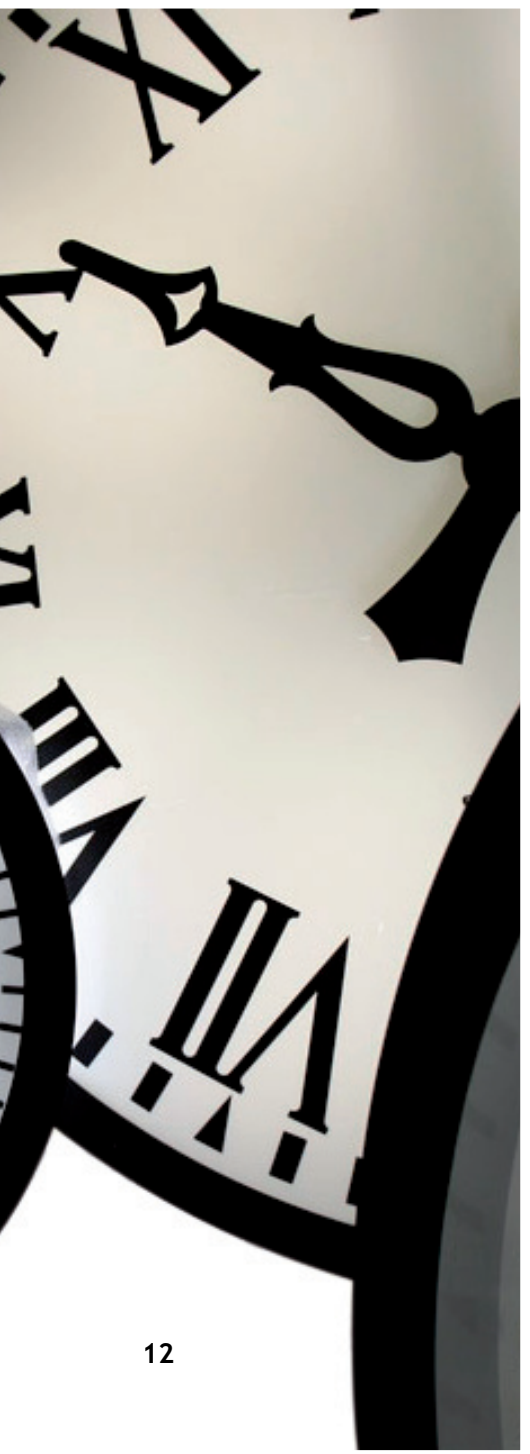

a los muertos en la tumba del pasado. Ese pasado ya ido y muerto es el pasado feudal del Antiguo Régimen, que no "está muy distante, en años, de nuestros ojos, pero que la Revolución lo oculta". El presente de esta "nueva sociedad" se transforma, entonces, en un pasaje entre un pasado terminado y un futuro del que, a pesar de su oscuridad, algunas verdades pueden discernirse.

Este sentimiento de ruptura con el pasado, que comparten no sólo los revolucionarios, sino, también, los reaccionarios se expresa hasta en la forma extrema de modificar el calendario. Esta manera que el "presente contemporáneo" tiene de oponerse al pasado - pasado que la Asamblea Nacional de Agosto de 1789 votó "destruir por completo"- es totalmente nueva.

Diversos elementos confluyeron para que los europeos del siglo XIX experimentaran su presente como contemporáneo. En primer lugar, el tiempo se asume como universal, es decir, se concibe que todo el mundo está atravesado por un único esquema cronológico que, a su vez, es lineal. Esta idea de universalidad cronológica está disponible a partir del siglo XVIII cuando Newton define al tiempo absoluto como el único "verdadero y matemático" que "fluye uniformemente sin relación a ninguna cosa externa". Este tiempo universal, lineal y cronológicamente mensurable se distingue tanto del tiempo universal como la suma de partes de Bossuet, como lo universal pensado como sistema en la historia del mundo de Kant o como lo universal del cristianismo que, en la llamada "Edad Media" europea convive con una tiempo latino, un tiempo griego y un tiempo árabe-musulmán.

El presente contemporáneo que el siglo XIX experimenta como época supone un tiempo universal que es incompatible con la coexistencia de otras posibles formas temporales. El presente contemporáneo se transforma en el "ahora" de un único marco temporal en el que todos los acontecimientos adquieren una posición. El continuo temporal puede recorrerse desde el pasado hacia el presente o desde el presente hacia el pasado. Esta idea se concreta cuando en 1884 se realiza, en Washington D.C., The International Meridian Conference a la que asistieron 41 delegados de 24 naciones. Entre sus muchos principios se estableció que "era deseable adoptar un único meridiano mundial para reemplazar a los muchos que coexisten y que todos los países debían adoptar un día universal". ${ }^{16}$ Si bien no todos los países lo aceptaron, revelando una lucha de poder para imponer un esquema temporal único, la introducción de un standard cronológico mundial fue condición de posibilidad para crear no sólo un único "recorrido" desde y hacia el presente sino, también, la posibilidad de concebir una simultaneidad temporal universal compartida.

En segundo lugar, si el tiempo se concibe como lineal y universal, la determinación temporal de un "presente contemporáneo" se realiza en relación al continuo temporal mismo. Organizar las épocas por medio de sucesiones dinásticas, o atendiendo, por ejemplo, a los metales o los libros sagrados, supone determinar al tiempo por medio de cualidades que expresarían características del período y que, de ningún modo dependen de un esquema temporal que presuponen. La perfección de las artes y la "grandeza" del espíritu humano es la cualidad que permite a Voltaire seleccionar sólo "cuatro siglos en la historia del mundo", entre los que, el siglo de Luis XIV es el "más feliz" de todos. Para Voltaire el siglo de Luis XIV es el "más ilustrado que haya existido jamás". La característica "per- 
fección de las artes y grandeza del espíritu humano" es la que determina, para Voltaire, la selección de estos siglos y no otros. Si la determinación temporal es diferente, dinastías, por ejemplo, o la "barbarie de los pueblos" para poner otro ejemplo, la selección de las épocas o períodos cambia también. Por el contrario, "contemporaneidad" es una peculiaridad que los propios europeos experimentan al ponerse, ellos mismos, en relación a un conjunto temporal a partir del que determinan un pasado ido y muerto frente al que experimentan su presente como un "novísimo tiempo". La determinación temporal no es independiente del continuo temporal. La novedad de lo contemporáneo sólo puede ser experimentada como tal, cuando se la relaciona con otros períodos que le anteceden y de los que difiere. La contemporaneidad, al transformarse en concepto de época, presupone una matriz temporal lineal en la que se posiciona como el último de los períodos. Por el contrario, el siglo de Luis XIV de Voltaire, por ejemplo, es una unidad en sí misma, para caracterizarlo no es necesario tener en cuenta ni al pasado (al siglo de "los Filipo y Alejandro" ni al de los "César y Augusto"), ni al futuro. El presente contemporáneo discrimina, necesariamente, el antes y el después, es decir, diacrónicamente. El presupuesto de universalidad del tiempo y el de determinación temporal "espacializan" al tiempo, es decir, el pasado de los muertos es el "atrás" del presente contemporáneo.

Sin embargo, si tomamos la matriz temporal lineal universal, no todos los que coexisten o viven simultáneamente a los europeos del siglo XIX, son sus contemporáneos. La contemporaneidad se delimita no sólo en relación al continuo temporal según el antes y el después, sino, también, y en tercer lugar, lo contemporáneo discrimina en el mismo presente: no todo lo coetáneo es contemporáneo. No todos comparten el mismo presente. Ser contemporáneo en el siglo XIX significa pertenecer a una clase homogénea cuya característica es haber dejado atrás a un pasado feudal. Los contemporáneos son los que habitan en el estado moderno. El estado moderno se transforma en norma política que permite discriminar en el presente a los "otros", que son aquellos que viven bajo otras normas políticas (los primitivos). Pero también el estado moderno permite discriminar retrospectivamente. El pasado sólo pertenece a los muertos que han contribuido a alcanzar el estado moderno, pero hay pueblos "sin historia" que si bien habitaron el pasado no fue aquel que contribuyó a alcanzar el presente contemporáneo.

Las otras naciones que coexisten o son simultáneas, cronológicamente hablando, a la Francia y Europa del siglo XIX, es decir, a Occidente, pueden no ser sus contemporáneas. En el siglo XIX ser "contemporáneo" y ser "occidental" son dos caras de la misma moneda (cronopolítica y geopolítica). Tal como lo expresa claramente Taine: aun cuando la eclosión del estado moderno es casi universal y simultánea, hay pueblos que están relegados pues no han salido del estado feudal, se encuentran "más atrasados". La contemporaneidad no sólo reconfigura al pasado, expulsándolo hacia "atrás" como "feudal y muerto" (discriminación diacrónica); sino que, también, reconfigura al presente diferenciando entre aquellos que no son mis contemporáneos por no compartir el mismo estadio político-cultural (discriminación sincrónica). La simultaneidad vital o coetaneidad temporal no garantiza la contemporaneidadad política-cultural. La cualificación de un tiempo lineal y universal produce, necesariamente una desincronización cualitativa. La experiencia epocal de contemporaneidad nace, en el 
siglo XIX, a partir de la exclusión de aquellos que no comparten un mismo presente político. La exclusión se da necesariamente porque la contemporaneidad no sólo crea una relación temporal, sino que ésta es resultado de una decisión normativa. El presente contemporáneo excluye al pasado como lo "otro": el "pasado histórico" es el resultado de esta operación diacrónica. Pero el presente contemporáneo también excluye a "otros" que viven en el mismo presente cronológico: los no-contemporáneos son los "otros" de esta operación sincrónica.

El presupuesto de universalidad permite insertar a todos en la misma línea del tiempo continuo y distingue al "pasado" del presente contemporáneo. El presupuesto de homogeneidad que introduce la norma sirve para diferenciar entre todos los coetáneos, es decir, aquellos que viven simultáneamente en el presente cronológico, a aquellos que son contemporáneos de aquellos que no lo son, y discriminar sincrónicamente. El "estado moderno" es el referente normativo que distingue, en el siglo XIX, a los contemporáneos de los que no lo son. La posición que se ocupa en el "espectro" es concebida en términos de "distancia": los "otros" están más o menos "atrasados" ("the rest") en relación al moderno estado contemporáneo ("the west"). La metáfora de la distancia temporal sólo es posible dentro de este tipo de matriz temporal normativa. Lo contemporáneo se transforma en el referente temporal normativo que permite medir la lejanía o cercanía diacrónica y sincrónica.

La antropología y la historia se instauran como saberes a fines del siglo XVIII y se profesionalizan durante el siglo XIX. El presente crea dos tipos de alteridades temporales a los que corresponden dos géneros de saberes con sus respectivos objetos. El "salvaje" es el que se encuentra no sólo geográficamente fuera de la Europa occidental sino que está "atrasado" en el tiempo, no es su contemporáneo, es un "primitivo". La distancia entre el salvaje primitivo y los civilizados contemporáneos es salvada por la antropología. La historia, por su parte, tiende un puente entre los muertos del Antiguo Régimen y el presente que se comprende como nuevo. Tanto la historia como la antropología son el resultado de estas acciones sobre el tiempo.

Ahora bien, ¿podemos evitar o impedir este tipo de políticas del tiempo que generan este tipo de alteridades a través de la exclusión de otros del presente? Es decir, ¿podemos evitar que el presente se constituya en referente sociocultural a partir del que se traza la diferencia sincrónica o diacrónica, es decir, un presente hegemónico? Creo que no. Siempre que la norma, (ya sea el estado moderno, la gloria de otros tiempos, la juventud perdida o el valor cultural que fuere) se introduzca en una cronología lineal que se pretende universal, inevitablemente el presente se construye como referente que discrimina al otro como "más atrasado", como "anacrónico", como no contemporáneo.

Texto recebido e aprovado em novembro de 2017. 